Abtsract P257 Table 1 Selected results

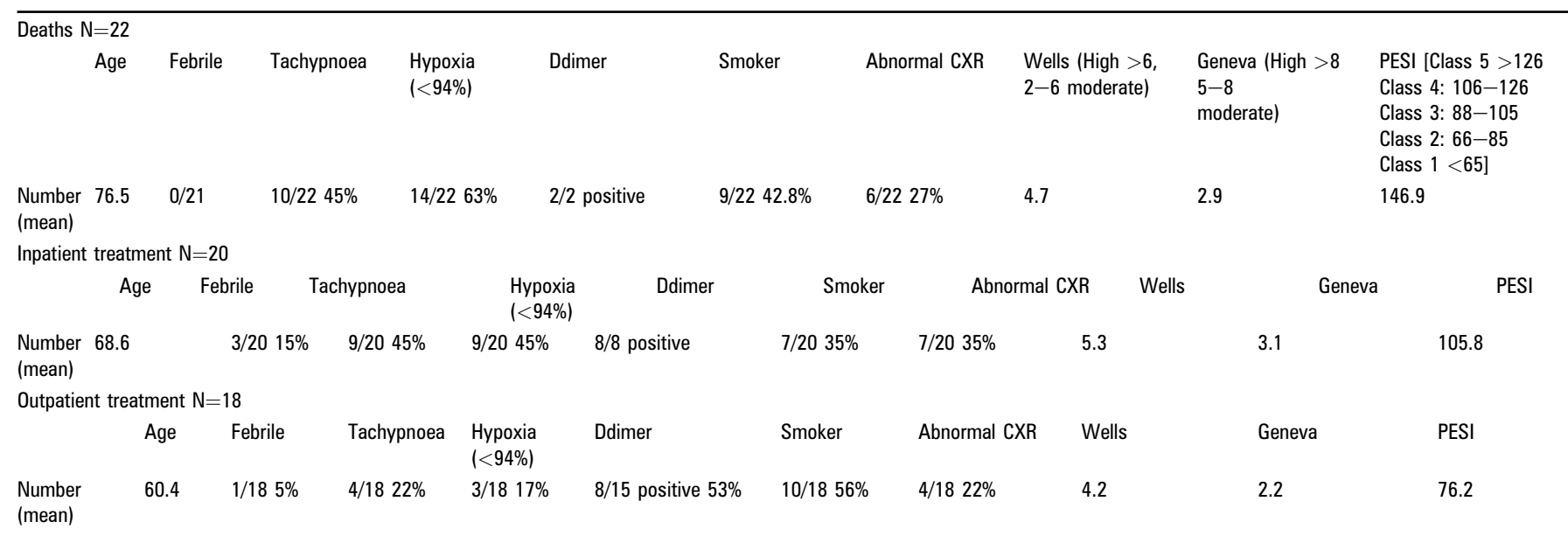

radiology and hypoxia. Managing $\mathrm{PE}$ as an outpatient potentially requires a combination of scoring systems in a prospective study.

\section{P258 IS CHRONIC THROMBOEMBOLIC PULMONARY HYPERTENSION (CTEPH) UNDER DIAGNOSED IN THE UK?}

doi:10.1136/thx.2010.151076.9

${ }^{1} \mathrm{~S}$ Sturney, ${ }^{1} \mathrm{G}$ Robinson, ${ }^{2} \mathrm{G}$ Coghlan, ${ }^{3} \mathrm{~J}$ Pepke-Zaba, ${ }^{1} \mathrm{~J}$ Easaw, ${ }^{1} \mathrm{~J}$ Suntharalingam. ${ }^{1}$ Royal United Hospital, Bath, UK; ${ }^{2}$ Royal Free Hospital, London, UK; ${ }^{3}$ Papworth Hospital, Cambridge, UK

Introduction Chronic Thromboembolic Pulmonary Hypertension $(\mathrm{CTEPH})$ is a rare but treatable condition. In selected cases Pulmonary Endarterectomy (PEA) surgery is potentially curative, with 80-85 procedures performed annually at a single national centre, Papworth Hospital. Previous studies suggest the disease may be under diagnosed.

Aim To identify whether developing a local Pulmonary Hypertension (PH) service improves the number of CTEPH cases diagnosed in a DGH setting.

Methods In 2005, a regional PH satellite service was set up at the Royal United Hospital (RUH), Bath, in conjunction with the Royal Free, London. The service aims to provide local access to PH-specific therapies at national standards of care. New cases of CTEPH are investigated with a number of investigations including right heart catheterisation and pulmonary angiography and then referred to Papworth Hospital's MDT to determine eligibility for surgery. This abstract examines the number of cases diagnosed in RUH's own catchment area following a rolling education programme targeting local physicians and radiologists.

Results Prior to 2007 no RUH patients had undergone PEA surgery. Since 2007, 12 patients have been diagnosed with CTEPH (7 male) with mean age 62.3 years (SD 12.1, range $37-72)$. Only $5(41.7 \%)$ had a documented history of previous thromboembolic disease. Referrals were received from a variety of sources including respiratory, vascular, rheumatology, gastroenterology, primary care, ENT, stroke physician and cardiology. Four (33.3\%) were referred as inpatients. At referral 33.3\% were NYHA class II, $41.7 \%$ class III and $25.0 \%$ class IV. Mean pulmonary haemodynamics were: RA $10.9 \mathrm{~mm} \mathrm{Hg}$ (6.4), mPAP $46.3 \mathrm{~mm} \mathrm{Hg}$ (13.1), PCWP $13.2 \mathrm{~mm} \mathrm{Hg}$ (4.4), CO 4.81/min (0.9), PVR 7.8WU (4.1). 8 have undergone or are awaiting surgery, 1 died of unrelated causes and 3 are being treated medically. (Abstract P258 Figure 1).

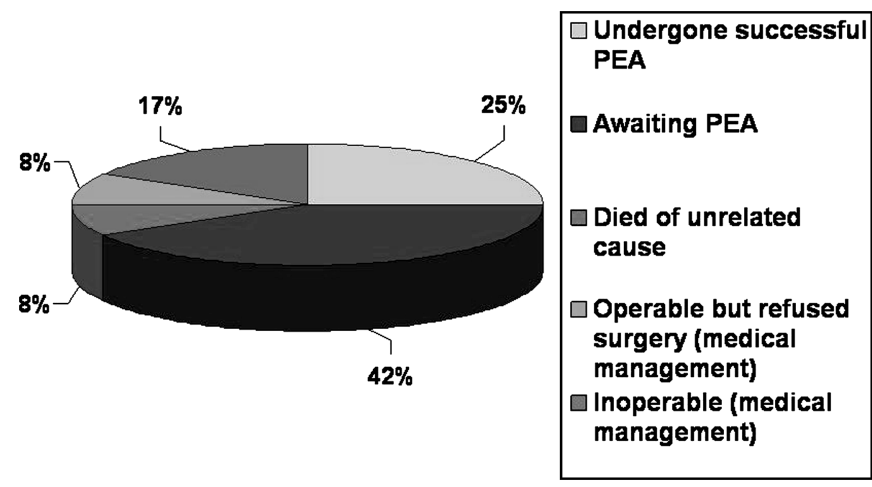

Abstract P258 Figure 1 Outcomes for patients with CTEPH diagnosed at RUH since 2007.

Conclusions Underdiagnosis of CTEPH may be exacerbated by patients presenting to a variety of specialties. The number of CTEPH cases diagnosed at the RUH has increased significantly since 2007, probably as a result of raised local awareness of the condition. It is therefore likely that this potentially curable condition is under diagnosed in areas with more limited access to $\mathrm{PH}$ specialist facilities.

\section{P259 INCIDENTAL FINDINGS ON CT PULMONARY ANGIOGRAPHY: WHAT SHOULD WE BE LOOKING FOR?}

doi:10.1136/thx.2010.151076.10

R Thiagarajah, M Qureshi, A Sahu, R Riordan. Peninsula Radiology Academy, Plymouth, UK

Introduction and Objectives The role of diagnostic imaging in pulmonary embolism (PE) is being increasingly undertaken by CT pulmonary angiography (CTPA) and there has been an explosion of usage in our trust in the last two years, which reflects a growing trend in clinical practice in the United Kingdom (UK). There has been an abundance of literature on how best to utilise CTPA with particular attention to optimisation of pulmonary artery opacification to aid diagnosis. An additional benefit of CTPA over other modalities for the diagnosis of PE that may be less widely appreciated is CTPAs ability to provide simultaneous information on other pathologies affecting the thorax and alternative diagnoses requiring further clinical management.

Methods To this cause a sample of 400 CTPAs were retrospectively reviewed in a 1001 bed acute teaching hospital in the UK, to identify 
the common types of additional pathology demonstrated. The presence of $\mathrm{PE}$ and any additional pathology, with special attention to "incidental" pathology, or pathology that was unsuspected but which was significant enough to change the patient's management was recorded, in order to identify the common incidental pathologies that we should be actively hunting for when reviewing a CTPA. Results PE was demonstrated in 94 studies $(22.6 \%$; $95 \%$ CI $18.7 \%$ to $26.9 \%$ ). Additional pathology was seen in 373 studies $(89.7 \%$; $95 \%$ CI $86.3 \%$ to $92.4 \%$ ), of which 58 (15.6\%; $95 \%$ CI $12.0 \%$ to $19.6 \%$ ) were reported as incidental. These included coronary calcification, degenerative spine, aortic disease, valve disease, liver cyst, retrosternal goitre, anatomical variant, gallstone/gallbladder disease, scapula lesion, rib fracture/deformity. Forty-three cases (10.3\%) went on to require further investigation or follow-up. One hundred and ten caes $(26.4 \%)$ showed no PE but an alternative diagnosis i.e. effusion/consolidation.

Conclusion The prevalence of PE in our sample was $22 \%$. Secondary findings were found in $89.7 \%$ of scans, with completely incidental findings reported in $15.6 \%$ and an alternative diagnosis when there was no PE in $26.4 \%$. This emphasises the usefulness of CTPA in supplying further diagnostic data in patients with suspected PE.

\section{P260 MANAGING THE INDETERMINATE CT PULMONARY ANGIOGRAM: DO WE GET IT RIGHT?}

doi:10.1136/thx.2010.151076.11

\section{E L O'Dowd, J D Birchall, R J Berg. Royal Derby Hospital, Derby, UK}

Introduction Around 5\% of CT pulmonary angiograms (CTPA) are indeterminate, usually due to incomplete contrast enhancement of pulmonary arteries, motion artefact or difficulty interpreting small peripheral filling defects. While BTS guidelines propose good quality negative CTPA as sufficient grounds to not treat for pulmonary embolism (PE), response to indeterminate CTPA is not addressed. No previous study has investigated variation in radiologists' reporting and clinicians' response to indeterminate CTPA's.

Methods Records of 51 patients with indeterminate CTPA were retrospectively reviewed for radiologist's reporting, clinicians' documentation of such, and clinical outcomes.

Results Reports included "no large central PE" ( $n=25)$, "no obvious PE within the limitations of the study" ( $n=14)$, "unable to exclude segmental/subsegmental PE" $(n=5)$, and "equivocal filling defects of uncertain significance" $(n=4)$. 17/51 (33\%) stated: "PE cannot be excluded." Further imaging was suggested in only 2 cases. Clinicians documented recognition of technical shortcomings, reported by radiologists, in only 16/51 cases (31\%), and recorded "no PE" in 29/51 (57\%). Clinicians assessed pre-test probability in 8/51 (8\%). 36 patients had the diagnosis of PE dismissed without further tests, of whom 26 were treated for other acute cardio-respiratory conditions revealed on CTPA. 0 had one or more additional tests (repeat CTPA, V/Q scan or Doppler $\mathrm{u} / \mathrm{s}$ ), confirming thrombo-embolic disease in 2. Further investigation was significantly more likely following scans stating "PE cannot be excluded" than all other reports; both overall (47\% vs $6 \%$, $\mathrm{p}<0.001$ ), and excluding patients with alternative acute cardio-respiratory diagnoses ( $66 \%$ vs $15 \%, \mathrm{p}<0.005) .3$ patients with indeterminate filling defects were anti-coagulated for PE. Of 2 patients with no filling defects reported, but anti-coagulated on grounds of clinical suspicion and indeterminate scan, 1 presented 2 weeks later with bleeding complications. Anticoagulation was discontinued when review and further investigation suggested PE was, in retrospect, unlikely. One untreated patient re-presented after 2 weeks with non-fatal PE.

Conclusion There is a lack of clear guidance, and considerable variation in radiological reporting of, and clinicians' response to indeterminate CTPA. Un-ambiguous reporting, repeat clinical assessment, appropriate consideration of alternative diagnoses, and further investigation where appropriate may reduce the risks of missed diagnosis or unwarranted anti-coagulation.

\section{P261 QUESTIONNAIRE TO ASSESS ACCEPTABILITY OF VENOUS THROMBOEMBOLISM RISK ASSESSMENT TOOL BY ADMITTING DOCTORS AND NURSES IN THE MEDICAL ASSESSMENT UNIT OF A DISTRICT GENERAL HOSPITAL}

doi:10.1136/thx.2010.151076.12

E K Nuttall, F Zaman. Royal Blackburn Hospital, Blackburn, UK

Introduction The House of Commons Health Committee estimated 25000 people in the UK die from preventable hospital acquired venous thromboembolism (VTE) every year. The Department of Health now state all adult patients must have documented VTE risk assessment and have given guidance on thrombosis and bleeding risk factors based on guidance from the National Institute of Clinical Excellence (NICE). Our hospital trust has raised awareness of appropriate prescribing of thromboprophylaxis by alerts on the local intranet, posters in clinical areas, at staff inductions and the development of a VTE risk assessment tool that will be included in the new generic assessment document. The tool was audited with thromboprophylaxis prescribing and found that none of the tools were filled in correctly and 20 out of 50 patients (40\%) were not prescribed thromboprophlaxis appropriately. We wanted to find out why these results occurred.

Method A questionnaire was given to 34 members of staff who are exposed to the new risk assessment forms; 16 doctors and 18 nurses.

Results All doctors were happy assessing VTE risk, whereas $61 \%$ of the nurses were not happy assessing all the risks included. The mean time to fill in the form was $4 \mathrm{~min}$ for doctors and nurses. $63 \%$ of doctors felt it should be a nursing job to fill in the risk assessment form whereas $77 \%$ of nurses felt it was a doctors job to complete the VTE risk assessment. 50\% of doctors stated they used the risk assessment form when prescribing thromboprophylaxis and $62 \%$ of doctors felt they didn't need a form to assess risk of VTE.

Conclusion The trust has used numerous ways to raise awareness of thromboprophylaxis prescribing, including the risk assessment tool. However, $40 \%$ of patients audited were not correctly prescribed thromboprophylaxis despite these measures. The majority of nurses felt it was a doctors job to fill in the tool as not all nurses were happy to assess all the risk factors. Only half the doctors stated they used the risk assessment tool and over half the doctors felt they didn't need a form to assess VTE risk. 